\title{
QCD effective charges from lattice data
}

\author{
A.C. Aguilar, ${ }^{a}$ D. Binosi ${ }^{b}$ and J. Papavassiliou ${ }^{c}$ \\ ${ }^{a}$ Federal University of $A B C, C C N H$, \\ Rua Santa Adélia 166, CEP 09210-170, Santo André, Brazil \\ ${ }^{b}$ European Centre for Theoretical Studies in Nuclear Physics and Related Areas (ECT*), \\ Villa Tambosi, Strada delle Tabarelle 286, I-38123 Villazzano (TN), Italy \\ ${ }^{c}$ Department of Theoretical Physics and IFIC, University of Valencia-CSIC, \\ E-46100, Valencia, Spain \\ E-mail: arlene.aguilar@ufabc.edu.br, binosi@ect.it, \\ joannis.papavassiliou@uv.es
}

ABSTRACT: We use recent lattice data on the gluon and ghost propagators, as well as the Kugo-Ojima function, in order to extract the non-perturbative behavior of two particular definitions of the QCD effective charge, one based on the pinch technique construction, and one obtained from the standard ghost-gluon vertex. The construction relies crucially on the definition of two dimensionful quantities, which are invariant under the renormalization group, and are built out of very particular combinations of the aforementioned Green's functions. The main non-perturbative feature of both effective charges, encoded in the infrared finiteness of the gluon propagator and ghost dressing function used in their definition, is the freezing at a common finite (non-vanishing) value, in agreement with a plethora of theoretical and phenomenological expectations. We discuss the sizable discrepancy between the freezing values obtained from the present lattice analysis and the corresponding estimates derived from several phenomenological studies, and attribute its origin to the difference in the gauges employed. A particular toy calculation suggests that the modifications induced to the non-perturbative gluon propagator by the gauge choice may indeed account for the observed deviation of the freezing values.

KEYwORDS: Nonperturbative Effects, QCD

ARXIV EPRINT: 1004.1105 


\section{Contents}

1 Introduction 1

2 The two effective charges: definitions and basic concepts 3

2.1 Effective charge from the ghost-gluon vertex 4

2.2 The PT effective charge 5

$\begin{array}{lll}3 & \text { Effective charges from lattice } & \mathbf{7}\end{array}$

3.1 The basic building blocks: gluon and ghost propagators from the lattice 8

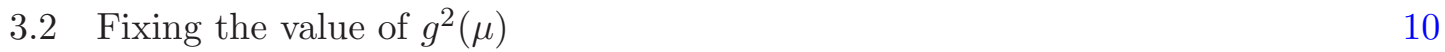

$3.3 G\left(q^{2}\right)$ and $L\left(q^{2}\right)$ : lattice data, renormalization subtleties, and SDE determination 12

$\begin{array}{llr}3.4 & \text { Final results } & 14\end{array}$

4 Reconciling lattice with phenomenology $\quad 15$

$\begin{array}{llr}5 & \text { Conclusions and outlook } & 18\end{array}$

\section{Introduction}

In recent years, a large number of independent lattice simulations have furnished highly non-trivial information on the infrared (IR) behavior of two fundamental ingredients of pure Yang-Mills theories, namely the (quenched) gluon and ghost propagators, for both $\mathrm{SU}(2)$ and $\mathrm{SU}(3)$ [1-6]. In particular, these simulations have firmly established that (in the Landau gauge) the QCD gluon propagator and the ghost dressing function are IR finite and non-vanishing. Given that the entire issue is under intense scrutiny, it is natural to explore some of the most salient theoretical and phenomenological implications of these lattice results. The purpose of the present work is to use the available lattice data to extract the running of the QCD effective charge for a wide range of physical momenta, and, in particular, its behavior and value in the deep IR. This quantity lies at the interface between perturbative and non-perturbative effects in QCD, providing a continuous interpolation between two physically distinct regimes: the deep ultraviolet (UV), where perturbation theory is reliable, and the deep IR, where non-perturbative techniques must be employed.

The generalization of the concept of the renormalization group (RG) invariant and process independent effective charge from QED to QCD is far from obvious, and has been discussed extensively in the literature. In this article we will consider two of the most standard definitions of the QCD effective charge. The first charge, to be denoted by $\alpha\left(q^{2}\right)$, constitutes the most direct non-Abelian generalization of the QED effective charge. This charge is obtained within the framework of the pinch technique (PT) [7-11], 
and its generalization, known as generalized PT (GPT), introduced in [12]. Of particular importance in this construction is the profound correspondence [13-17] that exists between the PT (GPT) and the background-field method (BFM) [18]. The second charge, to be denoted by $\alpha_{\text {gh }}\left(q^{2}\right)$, involves the ghost and gluon self-energies, in the Landau gauge, and in the kinematic configuration where the well-known Taylor non-renormalization theorem [19, 20] becomes applicable [21].

Both effective charges mentioned above display a strong dependence on the detailed characteristics of some of the most fundamental Green's functions of QCD. Specifically, in the case of $\alpha_{\mathrm{gh}}\left(q^{2}\right)$ the required ingredients are the conventional gluon propagator, $\Delta\left(q^{2}\right)$, (that of the $R_{\xi}$ gauges) and the ghost dressing function, $F\left(q^{2}\right)$; both quantities are simulated on the lattice, and we will use them as inputs for obtaining $\alpha_{\mathrm{gh}}\left(q^{2}\right)$. For $\alpha\left(q^{2}\right)$ the situation is slightly more involved. The fundamental ingredient one needs for obtaining $\alpha\left(q^{2}\right)$ is the gluon propagator of the PT-BFM, denoted by $\widehat{\Delta}\left(q^{2}\right)$, which, unfortunately, has not been simulated on the lattice yet. The way one establishes the required connection between the conventional gluon propagator (simulated on the lattice) and the PT-BFM propagator entering into the definition of $\alpha\left(q^{2}\right)$ is by resorting to two powerful non-perturbative identities. First, a formal relation known as "background-quantum" identity [22, 23], given in eq. (2.11), relates the two gluon propagators by means of a special function, $G\left(q^{2}\right)$, which plays a central role in the new Schwinger-Dyson equations (SDE) derived within the PT framework $[24,25]$. In fact, interestingly enough, in the Landau gauge only, $G\left(q^{2}\right)$ coincides with the well-known Kugo-Ojima function [26]. The second identity, given in eq. (2.16), allows one to obtain $G\left(q^{2}\right)$ from $F\left(q^{2}\right)$, to a very good approximation, given that the function that controls their difference, $L\left(q^{2}\right)$, is numerically rather small, and vanishes exactly at $q^{2}=0$. Therefore, even though the theoretical origin of these two effective charges is vastly different [e.g., $\alpha\left(q^{2}\right)$ originates from a propagator, while $\alpha_{\mathrm{gh}}\left(q^{2}\right)$ from a vertex], they are very close in the entire range of physical momenta, and exactly coincide in the deep IR [27].

A large number of theoretical and phenomenological studies, based on a-priori very distinct approaches $[8,28-33,35,36,38-41]$ support the notion of the "freezing" of the QCD running coupling in the deep IR. In fact, when the QCD charge is constant (non-vanishing!) in the IR, and the quark masses are ignored, QCD becomes conformally invariant. Therefore, as has been emphasized amply in the recent literature [42], the IR finiteness of the QCD effective charge constitutes a crucial requirement for the applicability of the powerful AdS/CFT correspondence [43].

As has been argued in numerous works, the IR finiteness of the effective charge and that of the gluon propagator are inextricably connected: they can be both traced back to the same phenomenon, namely the non-perturbative generation of a dynamical gluon mass, through the implementation of the Schwinger mechanism at the level of the SDE governing the gluon propagator [44]. Within the PT-BFM framework, the SDE solutions for the gluon self-energy, denoted by $\widehat{\Delta}\left(q^{2}\right)$, are used to form the RG-invariant combination $\widehat{d}\left(q^{2}\right)=$ $g^{2} \widehat{\Delta}\left(q^{2}\right)$ which, in turn, may be cast in the form $\widehat{d}^{-1}\left(q^{2}\right)=\left[q^{2}+m^{2}\left(q^{2}\right)\right]\left\{b \ln \left(\frac{q^{2}+4 m^{2}\left(q^{2}\right)}{\Lambda^{2}}\right)\right\}$, where $b$ is the first coefficient of the QCD $\beta$ function, and $\Lambda$ the QCD mass scale of a few hundred $\mathrm{MeV}$. The non-perturbative generalization of $\alpha\left(q^{2}\right)$, the QCD effective charge, is contained in the curly brackets; evidently, the $m^{2}\left(q^{2}\right)$ in the argument of the 
logarithm tames the Landau pole, and $\alpha\left(q^{2}\right)$ freezes at a finite value in the IR, namely $\alpha^{-1}(0)=b \ln \left(4 m^{2}(0) / \Lambda^{2}\right)$.

The IR finiteness of the effective charge obtained from the lattice data becomes manifest in the following way. First, one uses the available data for the gluon, the ghost, and the Kugo-Ojima function, to construct the lattice version of the corresponding dimensionful (mass dimension -2 ) RG-invariant quantity, denoted by $\widehat{d}\left(q^{2}\right)$ in the case of $\alpha\left(q^{2}\right.$ ) (as above), and $\widehat{r}\left(q^{2}\right)$ in the case of $\alpha_{\mathrm{gh}}\left(q^{2}\right)$ [see eq. (2.14) and eq. (2.3), respectively]. The next step is to extract from $\widehat{d}\left(q^{2}\right)$ and $\widehat{r}\left(q^{2}\right)$ a dimensionless quantity, which will correspond to the associated effective charge. Both RG-invariant quantities have the the gluon propagator, $\Delta\left(q^{2}\right)$, as a common ingredient. Given that $\Delta\left(q^{2}\right)$ is effectively massive in the IR, one should follow the standard procedure used for massive gauge bosons, such as the $W$ and the $Z$, namely factor out a massive "tree-level" propagator of the form $\left[q^{2}+m^{2}\left(q^{2}\right)\right]^{-1}$. The procedure outlined above guarantees the freezing of the resulting coupling at a finite (non-vanishing) value. If, instead, a $q^{-2}$ is factored out of the IR finite gluon propagator, one obtains (trivially) an effective charge that vanishes exactly as $q^{2}$ in the IR.

The article is organized as follows. In section II we briefly review the definitions of the two effective charges under study, and recall the fundamental identities, eq. (2.11) and eq. (2.16), which relate their ingredients. Section III contains the main results of this work. In particular, after reviewing some of the most important lattice results on the (Landau gauge) gluon and ghost propagators, we construct the QCD effective charges and determine their freezing value in the deep IR. In section IV we discuss the sizable discrepancy between the freezing values obtained in the previous section and those favored by a variety of phenomenological studies. We argue that the main reason for the observed discrepancy is the difference in the gauge used: while the $\alpha(0)$ extracted from the lattice corresponds to the BFM Landau gauge, the phenomenological constraints are almost exclusively obtained in the BFM Feynman gauge. We will then derive an approximate formula that relates the two, suggesting that the discrepancy may be indeed accounted for by the difference in gauge choices. Finally, in section V we will discuss our results and present our conclusions.

\section{The two effective charges: definitions and basic concepts}

Before introducing the definitions of the effective charges and some of the important concepts related to them, we establish the necessary notation. The gluon and ghost propagator will be defined as

$$
\begin{aligned}
\Delta_{\mu \nu}(q) & =-i\left[P_{\mu \nu}(q) \Delta\left(q^{2}\right)+\xi \frac{q_{\mu} q_{\nu}}{q^{4}}\right] \\
D\left(q^{2}\right) & =\frac{i F\left(q^{2}\right)}{q^{2}}
\end{aligned}
$$

where $\xi$ denotes the gauge-fixing parameter, and $P_{\mu \nu}(q)=g_{\mu \nu}-q_{\mu} q_{\nu} / q^{2}$ is the usual transverse projector. One has $\Delta^{-1}\left(q^{2}\right)=q^{2}+i \Pi\left(q^{2}\right)$, with $\Pi_{\mu \nu}(q)=P_{\mu \nu}(q) \Pi\left(q^{2}\right)$ the gluon self-energy; finally $F\left(q^{2}\right)$ is the so called ghost dressing function. 


\subsection{Effective charge from the ghost-gluon vertex}

A reasonable definition of the QCD effective charge may be obtained from the ghost-gluon vertex in the Landau gauge $[21,30]$. Exploiting the fact that, in this gauge, the ghost-gluon vertex does not get renormalized, one can construct the RG-invariant product

$$
\widehat{r}\left(q^{2}\right)=g^{2}\left(\mu^{2}\right) \Delta\left(q^{2}\right) F^{2}\left(q^{2}\right) .
$$

It should be noted, however, that away from the Landau gauge additional information on the form-factor of the ghost-gluon vertex must be supplemented, in order to define the RG-invariant quantity analogous to the $\widehat{r}\left(q^{2}\right)$ of $(2.3)$. This necessity, even though is not a limitation of principle, brings about several ambiguities; for example, the aforementioned vertex form-factor depends on two physical momenta, and a particular choice of the scale must be implemented, in order for the effective charge to be a function of a single momentum scale. In other words, one cannot obtain a universal definition of the charge, i.e., one that does not depend on the specific kinematic details of the vertex employed.

Since $\Delta\left(q^{2}\right)$ and $F\left(q^{2}\right)$ correspond exactly to the quantities measured directly on the lattice, the non-perturbative behaviour of $\widehat{r}\left(q^{2}\right)$ is fully determined, without any additional assumptions. However, the extraction of a dimensionless and IR finite quantity from $\widehat{r}\left(q^{2}\right)$ introduces a certain model-dependence, associated with the (yet unknown) dynamical details of the effective gluon mass. Specifically, from the $\widehat{r}\left(q^{2}\right)$ one defines the effective charge as

$$
4 \pi \alpha_{\mathrm{gh}}\left(q^{2}\right)=\left[q^{2}+m^{2}\left(q^{2}\right)\right] \widehat{r}\left(q^{2}\right),
$$

where $\alpha\left(\mu^{2}\right)=g^{2}\left(\mu^{2}\right) / 4 \pi$. The quantity $m\left(q^{2}\right)$ appearing in eq. (2.4) is the effective gluon mass, displaying a non-trivial dependence on the momentum transfer $q^{2}$, a common feature of dynamically generated masses. On general grounds, we have that in the deep IR, $m(0) \equiv m_{0}>0$, while in the deep UV, $m\left(q^{2}\right)$ is supposed to vanish. The exact way that $m\left(q^{2}\right)$ approaches zero in the deep UV is determined by the operator product expansion, and in particular by the gauge-invariant gluon condensate $\left\langle G^{2}\right\rangle=\left\langle 0\left|: G_{\mu \nu}^{a} G_{a}^{\mu \nu}:\right| 0\right\rangle$ of dimension four (no quarks were considered ${ }^{1}$ ) [47, 48]. Specifically, for asymptotic momenta, $m^{2}\left(q^{2}\right) \sim\left\langle G^{2}\right\rangle / q^{2}$, i.e. the gluon mass displays power-law running [49-51].

The first lattice determination of $m_{0}$ dates back to the work of Bernard $[52,53]$; there, $m_{0}$ was defined as one half the energy stored in the flux tube between adjoint sources at the crossover or screening distance, i.e. at the separation at which two dynamical gluons are created. More recently [54], $m_{0}$ has been extracted from the lattice simulation of the gluon propagator in the Landau gauge. These lattice studies suggest that $m_{0} \sim 400-600 \mathrm{MeV}$, in agreement with the values favored by a variety of phenomenological estimates [55-62].

Unfortunately, to date, there is no definitive lattice determination of the full momentum dependence of $m\left(q^{2}\right)$; for some recent attempts see [63, 64]. The main difficulty can be

\footnotetext{
${ }^{1}$ In addition to $\left\langle G^{2}\right\rangle$, another quantity that might be relevant to these considerations is the gaugeinvariant non-local condensate of dimension two, usually denoted by $\left\langle A_{\min }^{2}\right\rangle$, obtained through the minimization of $\int d^{4} x\left(A_{\mu}\right)^{2}$ over all gauge transformation [45]. Note that $\left\langle A_{\min }^{2}\right\rangle$ should not be confused with the local gauge-variant condensate of dimension two $\left\langle 0\left|: A_{\mu}^{a} A_{a}^{\mu}:\right| 0\right\rangle$ (see, e.g. [46]) which cannot appear in the operator product expansion of gauge-invariant quantities.
} 


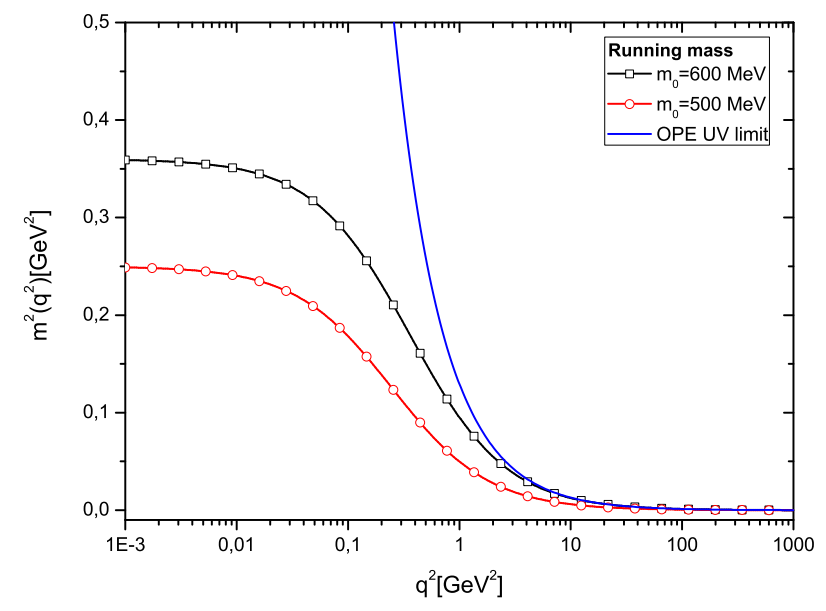

Figure 1. The dynamical gluon mass of eq. (2.5) with $m_{0}=600 \mathrm{MeV}$ (black curve with squares) and $m_{0}=500 \mathrm{MeV}$ (red curve with circles). The UV behavior predicted by operator product expansion is represented by the blue curve; clearly, it diverges in the IR.

traced back to the fact that the mass becomes very small in the UV, and its signal gets mixed in the background of the gluon propagator uncertainties. Therefore, for the purposes of the present work we will resort to a simple model of $m^{2}\left(q^{2}\right)$, which interpolates between the $m_{0}$ found on the lattice (deep IR) and the power-law running dictated by the operator product expansion (deep UV). Specifically, the functional form we will assume for $m^{2}\left(q^{2}\right)$ is given by

$$
m^{2}\left(q^{2}\right)=m_{0}^{4} /\left(q^{2}+m_{0}^{2}\right) ;
$$

evidently, the running mass of $(2.5)$ has a finite value at $q^{2} \rightarrow 0$, i.e. $m^{2}(0)=m_{0}^{2}$, with a power-law decrease in the deep UV. In figure 1 we show the dynamical gluon mass of eq. (2.5) for $m_{0}=600 \mathrm{MeV}$ and $m_{0}=500 \mathrm{MeV}$.

\subsection{The PT effective charge}

A universal (process-independent) definition of an effective charge for every gauge may be obtained from the gluon self-energy in the (covariant) BFM. As is well-known, this quantity, to be denoted by $\tilde{\Pi}^{\left(\xi_{Q}\right)}(q)$, captures the running of the QCD coupling for every value of the (quantum) gauge-fixing parameter, $\xi_{\mathrm{Q}}$. In particular, at one loop, we have

$$
i \tilde{\Pi}^{\left(\xi_{\mathrm{Q}}\right)}(q)=q^{2} g^{2}\left[b \ln \left(-q^{2} / \mu^{2}\right)+C_{\xi_{\mathrm{Q}}}\right],
$$

where $b=11 C_{\mathrm{A}} / 48 \pi^{2}$ is the first coefficient of the QCD $\beta$ function $\left(\beta=-b g^{3}\right)$ in the absence of quarks, $C_{\mathrm{A}}$ is the Casimir eigenvalue of the adjoint representation $\left(C_{\mathrm{A}}=N\right.$ for $\mathrm{SU}(N)$ ), and the gauge-dependent constant $C_{\xi_{\mathrm{Q}}}$ is given by (third item in [13-15])

$$
C_{\xi_{\mathrm{Q}}}=\frac{C_{\mathrm{A}}}{16 \pi^{2}}\left[\frac{\left(1-\xi_{\mathrm{Q}}\right)\left(7+\xi_{\mathrm{Q}}\right)}{4}-\frac{67}{9}\right] .
$$

Note that the value $\xi_{\mathrm{Q}}=1$, i.e., the Feynman gauge of the BFM, is very special, because it reproduces the (gauge-independent) PT gluon self-energy; in this privileged gauge all 
unphysical longitudinal terms appearing inside an ostensibly gauge-independent quantity (physical on-shell amplitude, Wilson-loop, etc) have been discarded.

For asymptotically large momenta one may neglect the constant $C_{\xi_{\mathrm{Q}}}$ next to the leading logarithm, and write, in any gauge, (Euclidean momenta)

$$
\widehat{\Delta}\left(q^{2}\right)=\frac{1}{q^{2}\left[1+b g^{2} \ln \left(q^{2} / \mu^{2}\right)\right]} .
$$

It is then easy to establish (e.g., by resorting to the QED-like identity $Z_{\widehat{A}}^{-1 / 2}=Z_{g}$, valid in the BFM to all orders and for every $\left.\xi_{\mathrm{Q}}[18]\right)$ that the product

$$
\widehat{d}\left(q^{2}\right)=g^{2}\left(\mu^{2}\right) \widehat{\Delta}\left(q^{2}\right),
$$

is invariant under the renormalization group, i.e., it is a RG-invariant quantity, just as the $\widehat{r}\left(q^{2}\right)$ in (2.3). From $\widehat{d}\left(q^{2}\right)$ one may extract the QCD effective charge exactly as in (2.4), namely

$$
4 \pi \alpha\left(q^{2}\right)=\left[q^{2}+m^{2}\left(q^{2}\right)\right] \widehat{d}\left(q^{2}\right) .
$$

In order to make contact between $\widehat{\Delta}\left(q^{2}\right)$ appearing in the definition of the RG-invariant product $\widehat{d}\left(q^{2}\right)$ and the conventional propagator $\Delta\left(q^{2}\right)$ simulated on the lattice (in the Landau gauge), we employ a formal all-order identity, which relates them as follows [22, 23]

$$
\Delta\left(q^{2}\right)=\left[1+G\left(q^{2}\right)\right]^{2} \widehat{\Delta}\left(q^{2}\right) .
$$

In the above formula the two gauge fixing constants, $\xi$ and $\xi_{Q}$, associated with $\Delta\left(q^{2}\right)$ and $\widehat{\Delta}\left(q^{2}\right)$, respectively, must be equal (but otherwise arbitrary); in particular, in the Landau gauge, $\xi=\xi_{\mathrm{Q}}=0$.

The function $G\left(q^{2}\right)$ appearing in (2.11) is the $g_{\mu \nu}$ component of a particular two-point function, denoted by $\Lambda_{\mu \nu}(q)$, defined as

$$
\begin{aligned}
\Lambda_{\mu \nu}(q) & =-i g^{2} C_{A} \int_{k} H_{\mu \rho}^{(0)} D(k+q) \Delta^{\rho \sigma}(k) H_{\sigma \nu}(k, q) \\
& =g_{\mu \nu} G\left(q^{2}\right)+\frac{q_{\mu} q_{\nu}}{q^{2}} L\left(q^{2}\right)
\end{aligned}
$$

where $\int_{k} \equiv \mu^{\epsilon}(2 \pi)^{-d} \int d^{d} k$, with $d=4-\epsilon$ the space-time dimension. The function $H_{\sigma \nu}(k, q)$ appears in the all-order Slavnov-Taylor identity satisfied by the three gluon vertex [65], and is related to the ghost-gluon vertex $\boldsymbol{\Gamma}_{\mu}(k, q)$ through the identity

$$
q^{\nu} H_{\mu \nu}(k, q)=-i \boldsymbol{\Gamma}_{\mu}(k, q) .
$$

At tree level, $H_{\mu \nu}^{(0)}=i g_{\mu \nu}$ and $\Gamma_{\mu}^{(0)}(k, q)=\Gamma_{\mu}(k, q)=-q_{\mu}$. Note that both $G\left(q^{2}\right)$ and $L\left(q^{2}\right)$ depend explicitly on the value of the gauge-fixing parameter $\xi$.

Since the origin of the identity in (2.11) is the BRST symmetry of the theory, it does not get deformed by the renormalization procedure. Thus, one can write (2.9) in terms of $\Delta\left(q^{2}\right)$ and $G\left(q^{2}\right)$ as follows

$$
\widehat{d}\left(q^{2}\right)=\frac{g^{2}\left(\mu^{2}\right) \Delta\left(q^{2}\right)}{\left[1+G\left(q^{2}\right)\right]^{2}}
$$


It turns out [66-68] that the function $G\left(q^{2}\right)$, coincides (in the Landau gauge only) with the well-known Kugo-Ojima function [26], $u\left(q^{2}\right)$ defined as

$$
\int d^{4} x \mathrm{e}^{-i q \cdot(x-y)}\left\langle T\left[\left(\mathcal{D}_{\mu} c\right)_{x}^{m}\left(\mathcal{D}_{\mu} \bar{c}\right)_{y}^{n}\right]\right\rangle=-\frac{q_{\mu} q_{\nu}}{q^{2}} \delta^{m n}+P_{\mu \nu}(q) \delta^{m n} u\left(q^{2}\right),
$$

where $\left(\mathcal{D}^{\mu} \Phi\right)^{m}=\partial^{\mu} \Phi^{m}+g f^{m n r} A_{\mu}^{n} \Phi^{r}$ is the usual QCD covariant derivative. The KugoOjima function has been simulated on the lattice by means of Monte-Carlo averages of the operator time-ordered product appearing on the left-hand side of the defining equation (2.15) [69-72]. Given that $G\left(q^{2}\right)=u\left(q^{2}\right)$, the lattice information on $u\left(q^{2}\right)$ may be used, in principle, into (2.14), together with the lattice results for the Landau gauge $\Delta\left(q^{2}\right)$. However, as we will explain in detail in subsection 3.3, the existing lattice results are afflicted by a certain ambiguity related to renormalization, a fact that limits their usefulness for the construction at hand.

In addition, $G\left(q^{2}\right)$ is related to the ghost dressing function $F\left(q^{2}\right)$ and the form-factor $L\left(q^{2}\right)$ of (2.12) through the BRST identity

$$
F^{-1}\left(q^{2}\right)=1+G\left(q^{2}\right)+L\left(q^{2}\right) .
$$

This identity, in conjunction with the corresponding dynamical equations [27] given in eq. (3.6), allows the indirect determination of $G\left(q^{2}\right)$ and $L\left(q^{2}\right)$ from the lattice data on the ghost dressing $F\left(q^{2}\right)$ [73]. Thus, provided that one carries out the renormalization procedure in a way that manifestly preserves (2.16), the two effective charges are related through the equation [27]

$$
\alpha_{\mathrm{gh}}\left(q^{2}\right)=\left[1+\frac{L\left(q^{2}\right)}{1+G\left(q^{2}\right)}\right]^{-2} \alpha\left(q^{2}\right) .
$$

An important corollary of the dynamical equations of eq. (3.6) is that $L(0)=0$. In addition, under very general conditions, $G(0) \in(-1,0)$. Therefore, from $(2.17)$ one concludes that $[27]$

$$
\alpha_{\mathrm{gh}}(0)=\alpha(0) .
$$

Since, finally, $\alpha_{\mathrm{gh}}\left(q^{2}\right)$ and $\alpha\left(q^{2}\right)$ coincide in the deep UV, where they both reproduce the correct perturbative behavior, the two charges can only differ appreciably in the intermediate region of momenta; however, since $L\left(q^{2}\right)$ is numerically suppressed [27], this difference is rather small.

\section{Effective charges from lattice}

This section contains the main results of this article, and is composed of several subsections. After presenting a collection of lattice data, which firmly establish the IR finiteness of the conventional gluon propagator $\Delta\left(q^{2}\right)$ (in the Landau gauge) and the ghost-dressing function, we embark on the actual extraction of the effective charges from the lattice data, using the definitions and results of the previous section. The final results of all the analysis, carried out throughout this section, are shown on the right panel of figure 9; evidently, in the deep IR, both charges, $\alpha_{\mathrm{gh}}\left(q^{2}\right)$ and $\alpha\left(q^{2}\right)$, saturate at the same finite value, as predicted on general principles. 

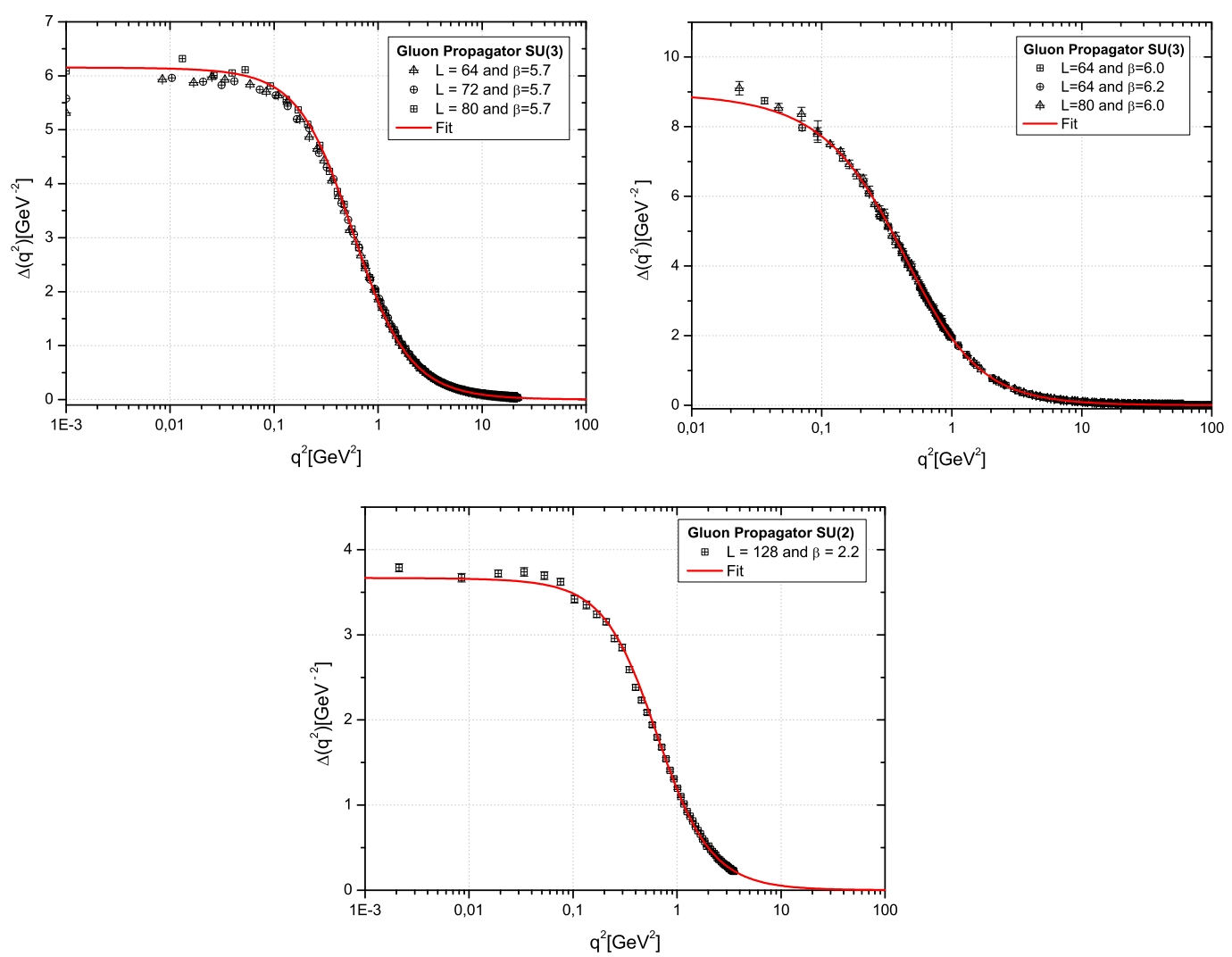

Figure 2. The lattice data for the gluon propagator obtained by three independent groups $[1,3,5,6]$ fitted by eq. (3.1). Upper left panel: Lattice data from ref. [3] renormalized at $\mu=3.0 \mathrm{GeV}$. Upper right panel: Lattice result, renormalized at $\mu=3.0 \mathrm{GeV}$, obtained in ref. [5, 6]. Bottom panel: The $\mathrm{SU}(2)$ gluon propagator obtained in ref. [1].

\subsection{The basic building blocks: gluon and ghost propagators from the lattice}

In this subsection we present some of the most relevant lattice results on the (Landau gauge) gluon propagator, given that it constitutes a central common ingredient of both effective charges. Even though in our analysis we will use only one set of lattice data (that of [3]), it is important to establish that various groups coincide on the qualitative behavior for the Green's functions in question. In figure 2 we show the results for the gluon propagator obtained by three independent lattice groups $[1,3,5,6]$. Although, for each group, the lattice spacing and the gauge group employed are different, all results have as a common feature the appearance of a plateau in the deep IR region, namely one of the most salient and distinctive predictions of the the gluon mass generation mechanism. In fact, the three set of data can be accurately fitted in terms of a massive gluon propagator of the type

$$
\Delta^{-1}\left(q^{2}\right)=m^{2}+q^{2}\left[1+\frac{13 C_{\mathrm{A}} g_{f}^{2}}{96 \pi^{2}} \ln \left(\frac{q^{2}+\rho m^{2}}{\mu^{2}}\right)\right],
$$

where $m^{2}, g_{f}^{2}$, and $\rho$ are treated as free fitting parameters. For the $\mathrm{SU}(3)$ lattice simulations, $\mu$ will be chosen to coincide with the renormalization point, while for the $\mathrm{SU}(2)$ case we will treat it as an adjustable parameter. 

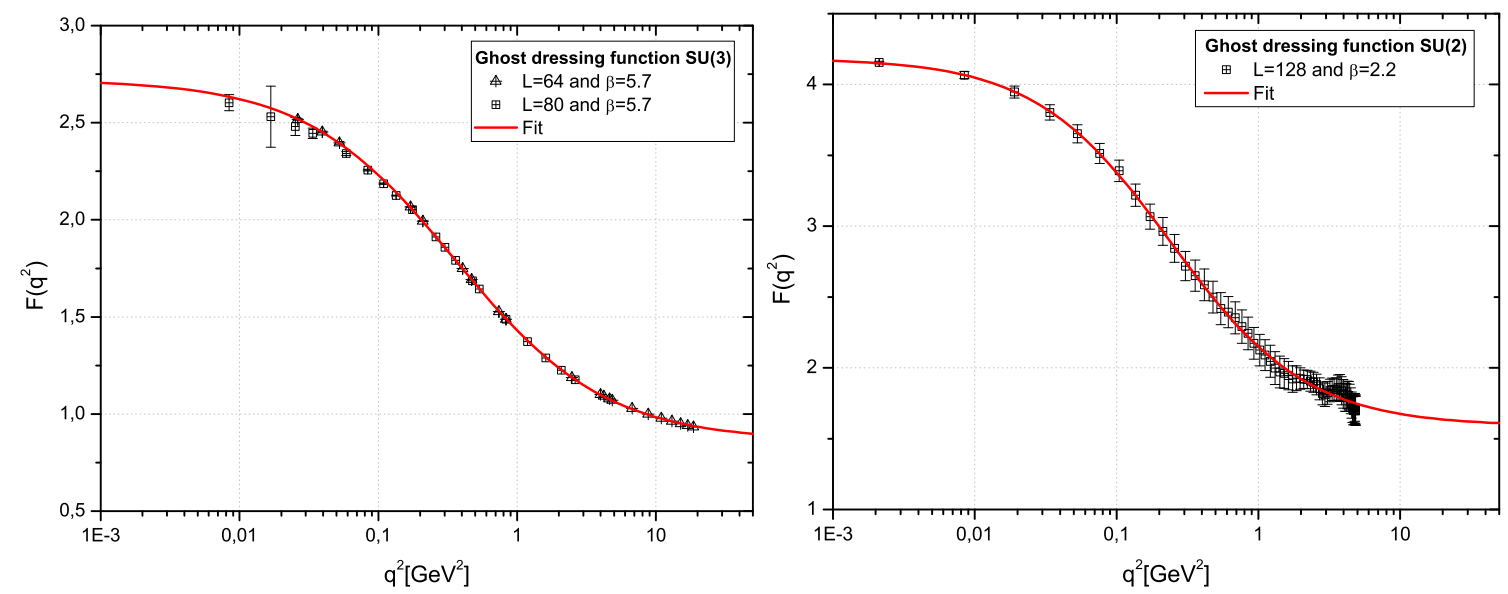

Figure 3. The lattice data for the ghost dressing function obtained by two independent groups $[1,3]$ fitted by eq. (3.2). Left panel: Lattice data from ref. [3] renormalized at $\mu=3.0 \mathrm{GeV}$. Right panel: The SU(2) ghost dressing function obtained in ref. [1].

Specifically, for the data presented on the upper left panel [3], we find that $m^{2}=0.16 \mathrm{GeV}^{2}, g_{f}^{2}=8.79, \rho=4$, and $\mu=3 \mathrm{GeV}$. For the upper right panel, we use $m^{2}=0.11 \mathrm{GeV} 2, g_{f}^{2}=9.77, \rho=9.6$, and $\mu=3 \mathrm{GeV}$; while the $\mathrm{SU}(2)$ lattice data of ref. [1] can be accurately adjusted using $m^{2}=0.27 \mathrm{GeV}^{2}, g_{f}^{2}=27.68, \rho=4$, and $\mu=1.92 \mathrm{GeV}$.

The parameter $m$ acts as a physical mass scale, whose function is to regulate the perturbative RG logarithm; so, instead of diverging at the Landau pole, the logarithm saturates at a finite value. Clearly, for large values of $q^{2}$, we recover the one-loop expression of the gluon propagator in the Landau gauge. Note also that for the purposes of this fit we have treated $m$ as if it were a hard mass, even though an important theoretical feature of the dynamically generated mass is that it should be function of the momentum, vanishing in the deep UV in a way consistent with the operator product expansion, [viz. eq. (2.5)].

In figure 3, we show the results for the ghost dressing function obtained by two independent lattice groups $[1,3]$. Both, $\mathrm{SU}(2)$ and $\mathrm{SU}(3)$ set of data, can be accurately fitted by the following expression

$$
F\left(q^{2}\right)=\frac{a_{1}-a_{2}}{1+\left(q^{2} / q_{1}^{2}\right)^{p_{1}}}+a_{2}
$$

The fit for the $\mathrm{SU}(3)$ ghost dressing function, presented on the left panel of figure 3, can be reproduced choosing $a_{1}=2.72, a_{2}=0.86, q_{1}^{2}=0.35$, and $p_{1}=0.80$; while for the $\mathrm{SU}(2)$ lattice data (right panel of figure 3 ), we use $a_{1}=4.18, a_{2}=1.59, q_{1}^{2}=0.24$, and $p_{1}=0.91$.

Notice that in the case of the ghost dressing function, the lattice data, and correspondingly our fit, show no enhancement in the deep IR; instead, $F\left(q^{2}\right)$ saturates at the constant value $a_{1}$.

Even though it is evident from figure 2 and figure 3 that the various lattice groups appear to be in qualitative agreement with each other, for the actual extraction the ef- 

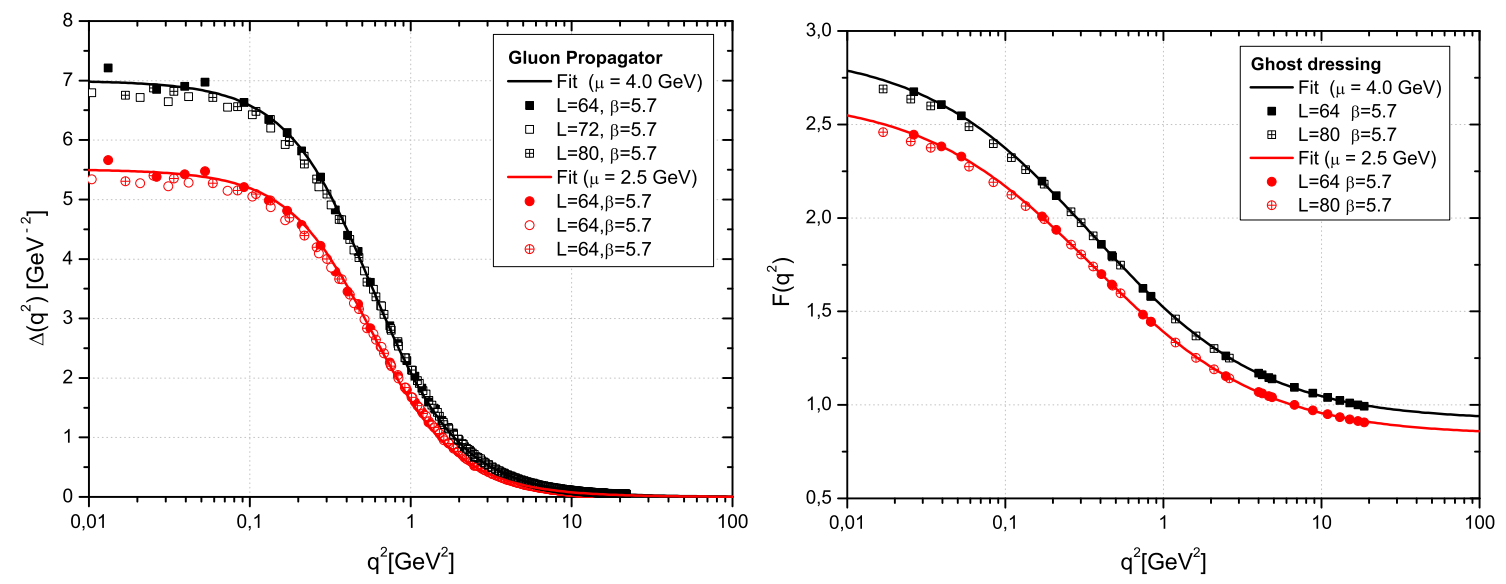

Figure 4. Lattice results for the gluon propagator (left panel), the ghost dressing function (right panel) renormalized at $\mu=2.5 \mathrm{GeV}$ (circles) and $\mu=4.0 \mathrm{GeV}$ (squares). The values of the fitting parameters of eqs. (3.1) and (3.2) are: $m^{2}=0.18 \mathrm{GeV}^{2}, g_{f}^{2}=10.64, a_{1}=2.64$, and $a_{2}=0.84$, for $\mu=2.5 \mathrm{GeV} ; m^{2}=0.14 \mathrm{GeV}^{2}, g_{f}^{2}=6.95, a_{1}=2.89$, and $a_{2}=0.91$, for $\mu=4.0 \mathrm{GeV}$. For both values of $\mu$ we use $\rho=4, p_{1}=0.8$, and $q_{1}^{2}=0.36 \mathrm{GeV}^{2}$.

fective charges we will rely on the data of ref. [3], given that this latter group uses SU(3) simulations, and has available data also on the ghost propagator.

In order to verify explicitly the expected $\mu$-independence of $\widehat{r}\left(q^{2}\right)$ and $\widehat{d}\left(q^{2}\right)$, we need to have at our disposal lattice data for $\Delta\left(q^{2}\right), F\left(q^{2}\right)$, and $G\left(q^{2}\right)$ at different renormalization points. To that end, we will exploit the property of multiplicative renormalizability, which allows one to connect a set of points renormalized at $\mu$ with the corresponding set renormalized at $\nu$, through the relations

$$
\Delta\left(q^{2}, \mu^{2}\right)=\frac{\Delta\left(q^{2}, \nu^{2}\right)}{\mu^{2} \Delta\left(\mu^{2}, \nu^{2}\right)}, \quad F\left(q^{2}, \mu^{2}\right)=\frac{F\left(q^{2}, \nu^{2}\right)}{F\left(\mu^{2}, \nu^{2}\right)} .
$$

Thus, choosing the two different values $\mu=2.5 \mathrm{GeV}$, and $\mu=4.0 \mathrm{GeV}$, we obtain the curves for $\Delta\left(q^{2}\right)$, and $F\left(q^{2}\right)$ shown in figure 4 .

\subsection{Fixing the value of $g^{2}(\mu)$}

The next step is to determine the value of the renormalized coupling $g^{2}\left(\mu^{2}\right)$ that enters in both the definitions of the two RG-invariant quantities, $\widehat{r}\left(q^{2}\right)$ and $\widehat{d}\left(q^{2}\right)$. To that end, we resort to the SDE equation governing $F\left(q^{2}\right)$, namely

$$
F^{-1}\left(q^{2}\right)=Z_{c}+g^{2} C_{\mathrm{A}} \int_{k}\left[1-\frac{(k \cdot q)^{2}}{k^{2} q^{2}}\right] \Delta(k) D(k+q),
$$

where $Z_{c}$ is determined by imposing the momentum subtraction (MOM) type of condition $F\left(\mu^{2}\right)=1$. The reason for using this particular dynamical equation is because it is given by a single "one-loop dressed" Feynman diagram, as opposed to the corresponding SDE for the gluon propagator, which involves a large number of one- and two-loop dressed diagrams, containing various fully dressed (and poorly studied) vertices. Note that the above SDE 

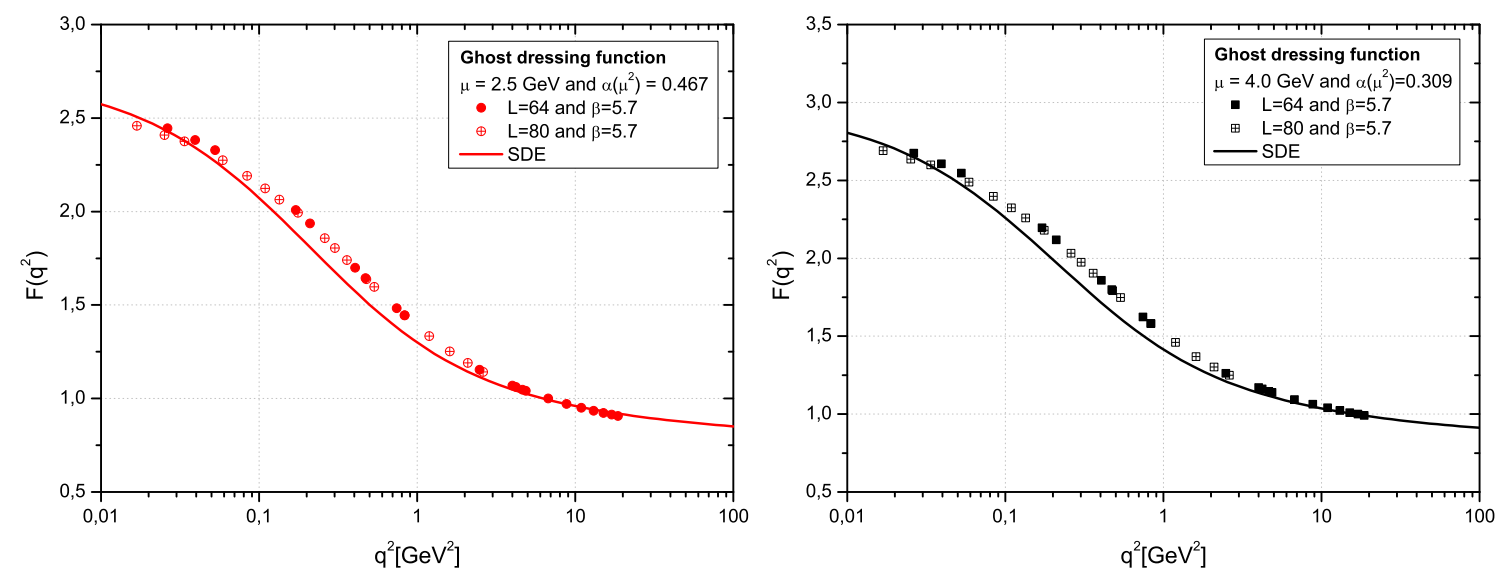

Figure 5. Comparison between the ghost dressing function $F\left(q^{2}\right)$ obtained from the ghost SDE (continuous lines) and the corresponding lattice data at $\mu=2.5 \mathrm{GeV}$ (left panel) and $\mu=4.0 \mathrm{GeV}$ (right panel).

has been derived using a tree-level expression for the fully dressed ghost-gluon vertex $\boldsymbol{\Gamma}_{\mu}$; this appears to be a good approximation, given that $\boldsymbol{\Gamma}_{\mu}$ has been studied in lattice simulations $[74,75]$, where it was found to deviate only mildly from its tree-level value.

The way the value of $g^{2}\left(\mu^{2}\right)$ is determined from eq. (3.6) is the following. One substitutes on the rhs of eq. (3.4) the lattice data for $\Delta\left(q^{2}\right)$, and solves the resulting integral equation for $F\left(q^{2}\right)$ numerically, adjusting the value of the $g^{2}\left(\mu^{2}\right)$ (multiplying the integral) such that the solution obtained coincides as well as possible with the available lattice data on $F\left(q^{2}\right)$.

The results of this procedure are presented in figure 5; in particular, we obtain the value $\alpha\left(\mu^{2}\right)=0.467$ for $\mu=2.5 \mathrm{GeV}$, and $\alpha\left(\mu^{2}\right)=0.309$ for $\mu=4.0 \mathrm{GeV}$.

The reason for quoting the values for $\alpha\left(\mu^{2}\right)$ with a three-decimal precision is because eq. (3.4) is extremely sensitive to any minor change in the value of $\alpha\left(\mu^{2}\right)$. In particular, increasing the value of $\alpha\left(\mu^{2}\right)$ by $1 \%$, which corresponds to a change in the third decimal of $\alpha\left(\mu^{2}\right)$, results in a $9 \%$ increase in the the value of $F(0)$.

The same procedure is repeated choosing renormalization points as deep in the UV as permited by the lattice data available. In particular, for $\mu=3.0 \mathrm{GeV}, \mu=3.3 \mathrm{GeV}$, $\mu=3.6 \mathrm{GeV}$, and $\mu=3.9 \mathrm{GeV}$, we obtain $\alpha\left(\mu^{2}\right)=0.395, \alpha\left(\mu^{2}\right)=0.356, \alpha\left(\mu^{2}\right)=0.335$, and $\alpha\left(\mu^{2}\right)=0.324$, respectively.

Then, in order to check if the $\alpha\left(\mu^{2}\right)$ values found through the above procedure are compatible with what one would expect within the MOM scheme that we use, we compare them with the corresponding four-loop perturbative calculation, presented in $[76,77]$. The result of this comparison is shown in figure 6 ; the yellow band is obtained by varying the $\Lambda_{\mathrm{QCD}}$, appearing in the expression derived in [76, 77], in the range between $350-450 \mathrm{MeV}$. As we can see, the best adjustment for the values of $\alpha\left(\mu^{2}\right)$ occurs for $\Lambda_{\mathrm{QCD}}=410 \mathrm{MeV}$. 


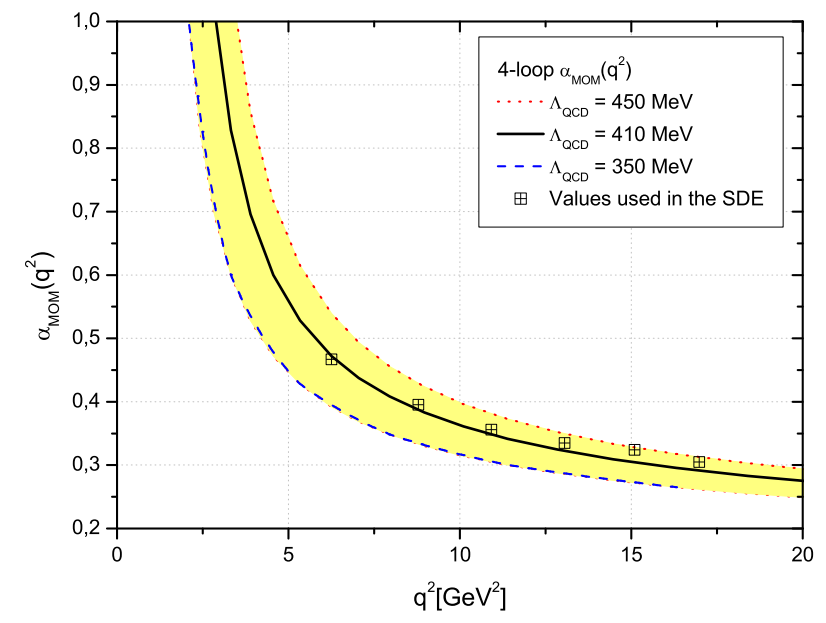

Figure 6. The perturbative running coupling in the MOM scheme, $\alpha_{\mathrm{MOM}}\left(q^{2}\right)$, up to four-loops for different values of $\Lambda_{\mathrm{QCD}}$. The black squares represent the values we use for $\alpha\left(\mu^{2}\right)$.

\section{3 $G\left(q^{2}\right)$ and $L\left(q^{2}\right)$ : lattice data, renormalization subtleties, and SDE deter- mination}

Whereas the RG-invariant quantity $\widehat{r}\left(q^{2}\right)$ defined from the ghost-gluon vertex needs as input for its determination the gluon propagator $\Delta\left(q^{2}\right)$ and the ghost dressing function $F\left(q^{2}\right)$, the corresponding PT quantity, $\widehat{d}\left(q^{2}\right)$ requires one additional ingredient, namely the function $G\left(q^{2}\right)$. As already mentioned in the previous section, $G\left(q^{2}\right)$ in the Landau gauge coincides with the KO function, usually denoted in the literature by $u\left(q^{2}\right)$. This function has been computed on the lattice in the early work of [69-71] and more recently in [72], mainly motivated by its relation with the well-known Kugo-Ojima confinement criterion. These lattice studies established clearly that the aforementioned criterion is not satisfied, since $u(0) \approx 0.6$ deviates appreciably from the special value of -1 .

The reason why the existing lattice data on $G\left(q^{2}\right)$ cannot be used in our analysis is rather subtle, and is directly related to the renormalization procedure, and in particular the fundamental identity of eq. (2.16). The difficulty lies in the fact that in [72] the renormalization of $G\left(q^{2}\right)$ was carried out without knowledge of the function $L\left(q^{2}\right)$. However, as has been explained in detail in [27], the self-consistent renormalization procedure of $G\left(q^{2}\right)$ requires the knowledge of $L\left(q^{2}\right)$. Specifically, in the MOM scheme the correct renormalization condition that preserves the validity of the crucial BRST identity of eq. (2.16) is

$$
G\left(\mu^{2}\right)=-L\left(\mu^{2}\right) .
$$

Given that $L\left(\mu^{2}\right) \neq 0$, the obvious implication of this condition is that one cannot choose simultaneously $F\left(\mu^{2}\right)=1$ and $G\left(\mu^{2}\right)=0$. Forcing $G\left(\mu^{2}\right)$ to assume a value other than that of (3.5) leads to a slight displacement of the entire $G\left(q^{2}\right)$ curve. To be sure, the error so introduced does not alter the important conclusion on the Kugo-Ojima confinement criterion, because, as we will see in a moment, $L\left(q^{2}\right)$ is rather small, so that it could not possibly account for the difference between the observed $u(0) \approx-0.6$ and the required $u(0)=-1$. However, for the purposes of the present work, it does distort the unique 

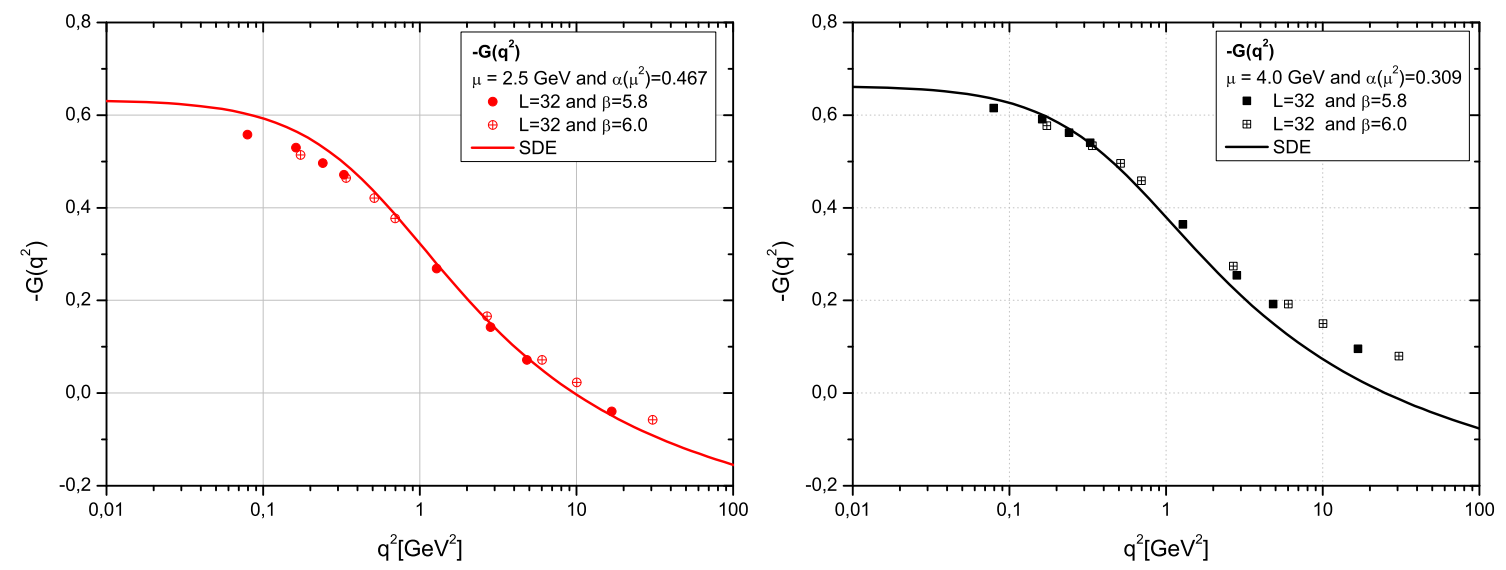

Figure 7. Comparison between the $G\left(q^{2}\right)$ function obtained from the SDE (continuous lines) and the corresponding lattice data at $\mu=2.5 \mathrm{GeV}$ (left panel) and $\mu=4.0 \mathrm{GeV}$ (right panel).

exact result available, namely that of eq. (2.18), which establishes the coincidence of two effective charges in the deep IR.

In order to circumvent this unpleasant artifact, we will appeal to the SDEs satisfied by $G\left(q^{2}\right)$ and $L\left(q^{2}\right)[27]$,

$$
\begin{aligned}
1+G\left(q^{2}\right) & =Z_{c}+\frac{g^{2} C_{\mathrm{A}}}{d-1} \int_{k}\left[(d-2)+\frac{(k \cdot q)^{2}}{k^{2} q^{2}}\right] \Delta(k) D(k+q), \\
L\left(q^{2}\right) & =\frac{g^{2} C_{\mathrm{A}}}{d-1} \int_{k}\left[1-d \frac{(k \cdot q)^{2}}{k^{2} q^{2}}\right] \Delta(k) D(k+q) .
\end{aligned}
$$

and will obtain $G\left(q^{2}\right)$ and $L\left(q^{2}\right)$ by substituting lattice data for $\Delta\left(q^{2}\right)$, the result for $F\left(q^{2}\right)$, and the values for $g^{2}\left(\mu^{2}\right)$ obtained in the previous subsection. Note that the above equations have been derived using tree-level value for the kernel $H_{\mu \nu}$ [viz. eq. (2.12)]. This is consistent with the corresponding approximation employed for deriving the ghost SDE of (3.4), given that $\boldsymbol{\Gamma}_{\mu}$ and $H_{\mu \nu}$ are connected by the identity (2.13).

The advantage of using the set of equations given in (3.6) is twofold. First, they have the important property $L(0)=0$ build in. Indeed, as was first established in [27], if $F\left(q^{2}\right)$ and $\Delta\left(q^{2}\right)$ are both IR finite, then

$$
\left.\int_{k}[1-d f(k, q)] \Delta(k) D(k+q)\right|_{q \rightarrow 0}=0
$$

and so, from the second of (3.6) follows immediately that $L(0)=0$. Second, once $F\left(\mu^{2}\right)=1$ has been imposed, the value of $G\left(\mu^{2}\right)$ is completely determined from its own equation, and is automatically in conformity with eq. (3.5).

Next, using as ingredients the data for $\Delta\left(q^{2}\right)$ and $F\left(q^{2}\right)$ presented in figure 4 and figure 5 respectively, and the values of $\alpha\left(\mu^{2}\right)$ quoted in figure 6 , we carry out the corresponding integration numerically, and obtain the functions $G\left(q^{2}\right)$ and $L\left(q^{2}\right)$.

The comparison of SDE result for $G\left(q^{2}\right)$ with the lattice data obtained in [72] are shown in figure 7 for both values of $\mu^{2}$. As we can see, the numerical results obtained 


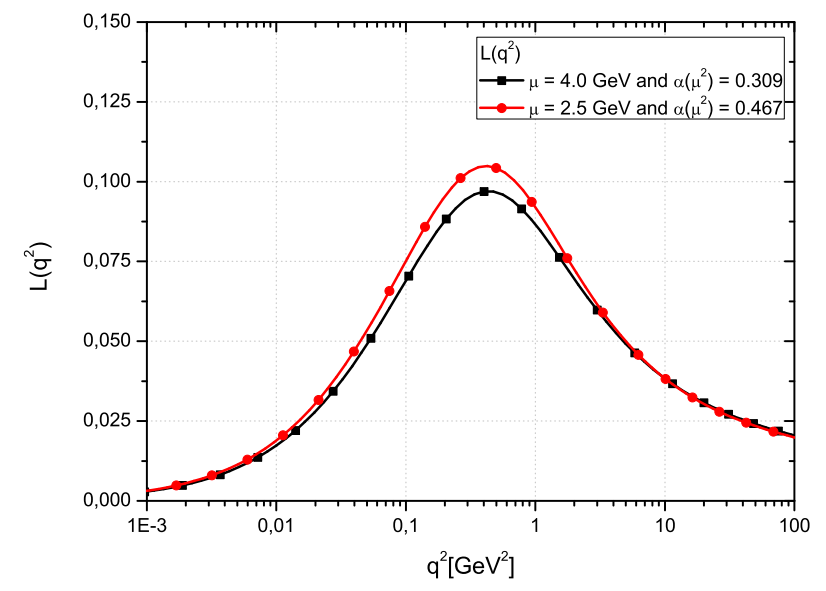

Figure 8. $L\left(q^{2}\right)$ determined from the corresponding $\operatorname{SDE}(3.6)$, using the solutions for $\Delta\left(q^{2}\right)$ and $F\left(q^{2}\right)$ presented in the figure 4 and figure 5 respectively, at the same renormalization point.

with eq. (3.6) are in good agreement with the lattice data. However, notice that there is a small discrepancy in the UV region due to the fact that the lattice data do not respect the renormalizatin condition given in eq. (3.5); instead, the result obtained through eq. (3.6) does. More specifically, for $\mu=2.5 \mathrm{GeV}$, eq. (3.6) gives $G\left(\mu^{2}\right)=-L\left(\mu^{2}\right)=-0.046$; while for $\mu=4.0 \mathrm{GeV}, G\left(\mu^{2}\right)=-L\left(\mu^{2}\right)=-0.033$.

The results for $L\left(q^{2}\right)$ are presented in figure 8 for both values of $\mu^{2}$. From figure 8 it is easy to check the three properties of $L\left(q^{2}\right)$ mentioned before: (i) indeed $L\left(q^{2}\right)$ is numerically rather small over the full range of momenta, (ii) it vanishes in the deep IR, and (iii) its maximum occurs in the intermediate momenta region (around $500 \mathrm{MeV}$ ).

\subsection{Final results}

From all the ingredients presented so far, one may construct the two RG-invariant quantities, $\widehat{r}\left(q^{2}\right)$ and $\widehat{d}\left(q^{2}\right)$ of eqs. (2.3) and (2.14). A crucial check of the self-consistency of the entire procedure is the numerical verification of the theoretically expected independence of the above quantities of the renormalization point $\mu$. To verify this important point, $\widehat{r}\left(q^{2}\right)$ and $\widehat{d}\left(q^{2}\right)$ have been calculated using into the defining equations two different sets of inputs for $\Delta, F$, and $G$, one set renormalized at $\mu=4.0 \mathrm{GeV}$, and another renormalized at $\mu=2.5 \mathrm{GeV}$. The values for $\alpha\left(\mu^{2}\right)$ are precisely those obtained through the procedure of the previous subsection, namely $\alpha\left(\mu^{2}\right)=0.467$ for $\mu=2.5 \mathrm{GeV}$, and $\alpha\left(\mu^{2}\right)=0.309$ for $\mu=4.0 \mathrm{GeV}$. The results of this construction are shown on the left panel of figure 9; clearly, the $\widehat{r}\left(q^{2}\right)$ and $\widehat{d}\left(q^{2}\right)$ obtained from each set of data are practically on top of each other, thus numerically confirming the theoretical expectations. One can also see that the two quantities behave as expected, differing only in the intermediate region of momenta $(20-600 \mathrm{MeV})$.

At this point, the non-perturbative running charges, $\alpha_{\mathrm{gh}}\left(q^{2}\right)$, and $\alpha\left(q^{2}\right)$, defined in eqs. (2.4) and (2.10), respectively, may be extracted by multiplying the results obtained for the corresponding RG-invariant quantities by the factor $\left[q^{2}+m^{2}\left(q^{2}\right)\right]$. To do that, 

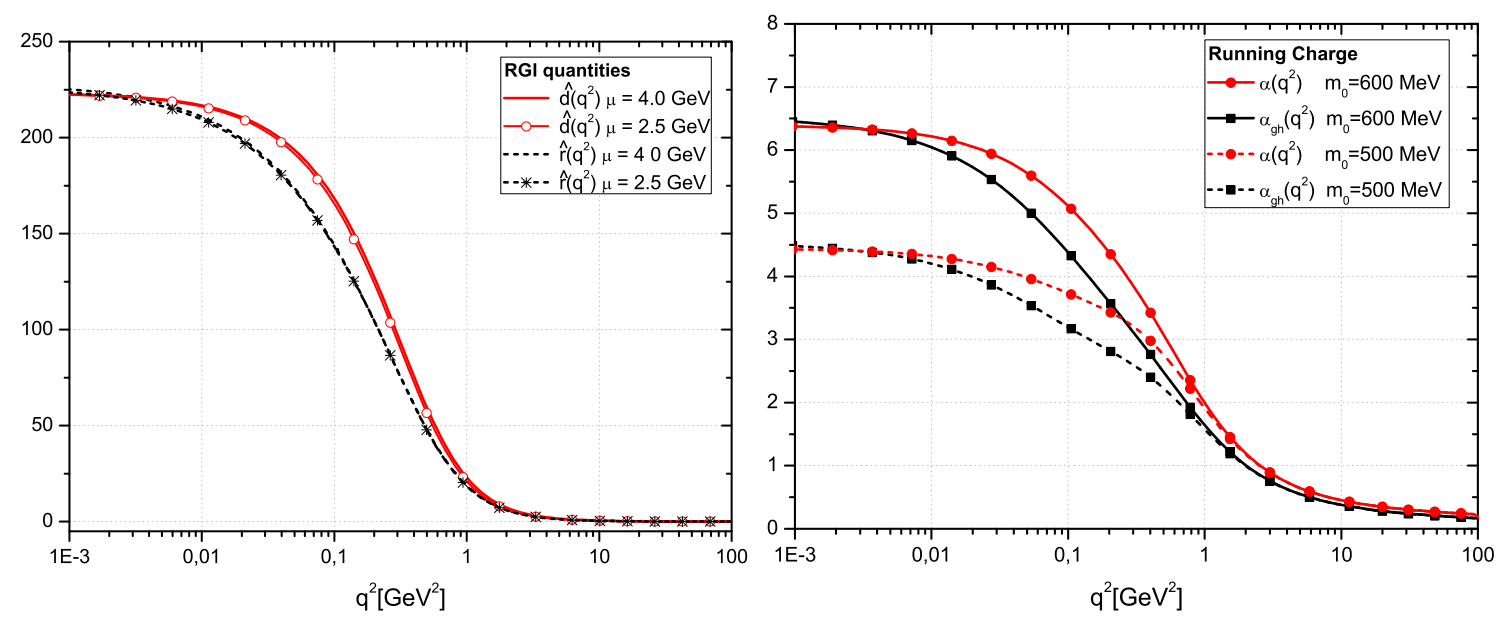

Figure 9. Left panel: Comparison between the two RG-invariant products $\widehat{d}\left(q^{2}\right)$ (solid line) and $\widehat{r}\left(q^{2}\right)$ (dashed line); notice that there are two overlapping curves at different $\mu$ for each product. Right panel: Comparison between the QCD effective charge extracted from lattice data: $\alpha\left(q^{2}\right)$ (red line with circles) and $\alpha_{\mathrm{gh}}\left(q^{2}\right)$ (black line with squares) for two different masses: $m_{0}=500 \mathrm{MeV}$ (dashed) and $m_{0}=600 \mathrm{MeV}$ (solid).

however, one must assume a functional form for the running mass $m^{2}\left(q^{2}\right)$; we will use the mass given in eq. (2.5).

The effective charges obtained following the above steps are shown in the right panel of figure 9. Evidently, both charges exhibit the correct (UV) perturbative behavior, and freeze at the same finite IR values corresponding to $\alpha_{\mathrm{gh}}(0)=\alpha(0)=4.45\left(m_{0}=500 \mathrm{MeV}\right)$ and $\alpha_{\mathrm{gh}}(0)=\alpha(0)=6.40\left(m_{0}=600 \mathrm{MeV}\right)$. The difference between the two couplings is only in the intermediate momenta region, and it is entirely due to the $L\left(q^{2}\right)$ function; in this region $\alpha\left(q^{2}\right)$ is always bigger than $\alpha_{\mathrm{gh}}\left(q^{2}\right)$.

\section{Reconciling lattice with phenomenology}

The effective charges we have obtained from the lattice (within the MOM renormalization scheme that we use), reach values in the deep IR that are almost an order of magnitude higher than those obtained from a large number of phenomenological studies. In particular, while the charge obtained from the lattice ranges between $4.5-6.5$ [depending on the value of $m(0)$ ], the systematic fitting of numerous processes suggests values for the effective charge in the range $0.7 \pm 0.3$ (for a similar range of gluon masses).

If one were to take both lattice and phenomenological results at face value, one should attempt to determine the reason for this sizable discrepancy. In this section we will address this issue in the context of a toy calculation, and we will argue that the observed discrepancy may be traced back to the difference in the gauge used when extracting the lattice results (the Landau gauge of the BFM, $\xi_{\mathrm{Q}}=0$ ) and that assumed in the phenomenological studies (the Feynman gauge of the BFM, $\xi_{\mathrm{Q}}=1$ ). Even though we cannot reach firm conclusions, our calculation seems to indicate that the difference in the gauges may indeed reconcile lattice with phenomenology. 
The crux of the matter is that the effective charge entering into physical processes is neither $\alpha_{\mathrm{gh}}\left(q^{2}\right)$ nor $\alpha\left(q^{2}\right)$, but rather the genuine PT effective charge [7-11], to be denoted by $\alpha_{\mathrm{PT}}\left(q^{2}\right)$. This charge is defined exactly as $\alpha\left(q^{2}\right)$ in (2.10), but with the crucial difference that the propagator used to form the $\widehat{d}\left(q^{2}\right)$ is the PT gluon propagator, i.e., the BFM propagator calculated in the Feynman gauge. The Feynman gauge of the BFM is privileged, in the sense that it is selected dynamically when the gluon self-energy is embedded into a physical observable (such as an on-shell test-amplitude). Specifically, this gauge captures the net propagator-like subamplitude emerging after QED-like properties have been replicated inside the test-amplitude, by means of the PT procedure. Therefore, any gauge-related exchanges between the Green's functions put together to form observables, are eliminated in this particular gauge. Instead, the gluon propagator in the Landau gauge, for example, contains still residual unphysical contributions, which, when introduced into a physical amplitude, will cancel against similar terms from vertex and box diagrams (see third item in [13-15]).

For asymptotically large momenta the numerical difference between the charges defined in either gauge is controlled by the constant $C_{\xi_{\mathrm{Q}}}$, given in (2.7). Evidently, in the UV this difference is subleading, and cannot give rise to any appreciable difference. Nonperturbatively, however, the difference between the two charges may be sizable. If, for example, we subscribe to the notion of dynamical mass generation, a difference in the gauge may lead to a vastly different IR behavior. In order to gain a quantitative understanding of how the difference in the gauge used may cause a significant disparity in the infrared values of the corresponding effective charges, we consider a model where the gauge bosons are endowed with a mass at tree-level. This will allow us to calculate, at one-loop level, the deviation between the two propagators, and the discrepancy that it induces to $\alpha(0)$ and $\alpha_{\mathrm{PT}}(0)$. The model in question is simply the electroweak sector of the Standard Model, with the electric charge set to zero, or, equivalently, with $\sin \theta_{\mathrm{w}}=0$, where $\theta_{\mathrm{w}}$ is the electroweak mixing (Weinberg) angle. In this limit the three gauge bosons (two $W$ s and one $Z$ ) are degenerate.

At one-loop, the SU(2) gluon self-energy, or equivalently, the $Z$-boson self-energy, may be obtained from the results of [80], for any value of $\xi_{Q}$. Specifically, one has (in Minkowski space)

$$
\begin{aligned}
\widehat{\Pi}_{\xi_{Q}}\left(q^{2}\right)= & \Pi_{\mathrm{F}}\left(q^{2}\right)+\frac{g^{2}}{4(4 \pi)^{2}} \frac{q^{2}-m^{2}}{(1-d) m^{4}} H_{\xi_{Q}}\left(q^{2}\right), \\
H_{\xi_{Q}}\left(q^{2}\right)= & \frac{2 m^{2}}{q^{2}}\left[m^{2}+(9-4 d) q^{2}-\left(q^{2}+m^{2}\right) \xi_{\mathrm{Q}}\right]\left[A_{0}\left(\xi_{\mathrm{Q}} m^{2}\right)-A_{0}\left(m^{2}\right)\right] \\
& -\left[4(5-2 d) m^{4}-8(2-d) m^{2} q^{2}+m^{2} q^{2}+q^{4}\right] B_{0}\left(q^{2} ; m^{2}, m^{2}\right) \\
& +2 \frac{q^{2}+m^{2}}{q^{2}}\left[\left(1-\xi_{\mathrm{Q}}\right)^{2} m^{4}-2\left(3-2 d+\xi_{\mathrm{Q}}\right) m^{2} q^{2}+q^{4}\right] B_{0}\left(q^{2} ; m^{2}, \xi_{\mathrm{Q}} m^{2}\right) \\
& -\left(q^{2}+5 m^{2}\right)\left(q^{2}-4 \xi_{\mathrm{Q}} m^{2}\right) B_{0}\left(q^{2} ; \xi_{\mathrm{Q}} m^{2}, \xi_{\mathrm{Q}} m^{2}\right),
\end{aligned}
$$

where $\Pi_{\mathrm{F}}\left(q^{2}\right) \equiv \Pi_{(\xi=1)}\left(q^{2}\right)$ is the conventional gluon self-energy in the Feynman gauge, $m$ 
denotes the effective gauge boson mass, and $A_{0}$ and $B_{0}$ are given by

$$
\begin{aligned}
A_{0}\left(m^{2}\right) & =16 \pi^{2} \int_{k} \frac{1}{k^{2}-m^{2}}, \\
B_{0}\left(q^{2} ; m_{1}^{2}, m_{2}^{2}\right) & =16 \pi^{2} \int_{k} \frac{1}{\left(k^{2}-m_{1}^{2}\right)\left[(k+q)^{2}-m_{2}^{2}\right]} .
\end{aligned}
$$

Setting $\xi_{\mathrm{Q}}=1$ in the above formula we recover the standard PT result for the one-loop self-energy of the $Z$-boson $[81,82]$, to be denoted by $\widehat{\Pi}_{\mathrm{F}}\left(q^{2}\right)$, namely

$$
\widehat{\Pi}_{\mathrm{F}}\left(q^{2}\right)=\Pi_{\mathrm{F}}\left(q^{2}\right)-\frac{g^{2}}{4 \pi^{2}}\left(q^{2}-m^{2}\right) B_{0}\left(q^{2} ; m^{2}, m^{2}\right) .
$$

Let us now take the difference $R\left(q^{2}\right)$ between $\widehat{\Pi}_{\xi_{Q}}\left(q^{2}\right)$ calculated in the Landau and Feynman gauges $\left(\xi_{Q}=0\right.$, and $\xi_{Q}=1$, respectively); denoting the former by $\widehat{\Pi}_{\mathrm{L}}\left(q^{2}\right)$, one has in the limit $q^{2} \rightarrow 0$ and $d=4$,

$$
\begin{aligned}
R(0) & \equiv \widehat{\Pi}_{\mathrm{L}}(0)-\widehat{\Pi}_{\mathrm{F}}(0) \\
& =\left.\frac{g^{2}}{(4 \pi)^{2}}\left\{\frac{m^{4}}{6} \frac{\partial}{\partial q^{2}} B_{0}\left(q^{2} ; m^{2}, 0\right)+3\left[B_{0}\left(q^{2} ; m^{2}, 0\right)-B_{0}\left(q^{2} ; m^{2}, m^{2}\right)\right]\right\}\right|_{q^{2}=0}
\end{aligned}
$$

We next extend the one-loop expression given in (4.4) to the non-perturbative regime, by introducing the following approximations: (i) we replace the (tree-level) massive propagators appearing in the function $B_{0}$ by their fully dressed counterpart $\Delta$ (in Landau gauge), and (ii) the "hard" mass $m$ by its running counterpart. Then we find (in Euclidean space)

$$
\begin{aligned}
\left.\frac{\partial}{\partial q^{2}} B_{0}\left(q^{2} ; m^{2}, 0\right)\right|_{q^{2}=0} & \rightarrow-\left.\frac{1}{\pi^{2}} \frac{\partial}{\partial q^{2}} \int_{k} \frac{\Delta\left(k^{2}\right)}{(k+q)^{2}}\right|_{q^{2}=0}=\frac{1}{2} \Delta(0), \\
B_{0}\left(q^{2} ; m^{2}, 0\right)-\left.B_{0}\left(q^{2} ; m^{2}, m^{2}\right)\right|_{q^{2}=0} & \rightarrow \frac{1}{\pi^{2}} \int_{k} \frac{m^{2}\left(k^{2}\right) \Delta^{2}\left(k^{2}\right)}{k^{2}}
\end{aligned}
$$

Thus, in the $\mathrm{SU}(3)$ case one obtains the final result (with $y=k^{2}$ )

$$
R(0)=\frac{3}{2} \frac{\alpha\left(\mu^{2}\right) m_{0}^{2}}{4 \pi}\left[\frac{m_{0}^{2}}{12} \Delta(0)+3 \int d y m^{2}(y) \Delta^{2}(y)\right]
$$

where the multiplicative factor of $3 / 2$ corresponds to the ratio of the Casimir eigenvalues for the adjoint representations of the gauge groups $\mathrm{SU}(3)$ and $\mathrm{SU}(2)$.

Since in Euclidean space $R\left(q^{2}\right)$ changes sign, we obtain ${ }^{2}$

$$
\begin{aligned}
\widehat{\Delta}_{\mathrm{F}}\left(q^{2}\right) & =\frac{1}{q^{2}+\widehat{\Pi}_{\mathrm{F}}\left(q^{2}\right)}=\frac{1}{\left(q^{2}+\widehat{\Pi}_{\mathrm{L}}\left(q^{2}\right)\right)\left(1+\frac{R\left(q^{2}\right)}{q^{2}+\widehat{\Pi}_{\mathrm{L}}\left(q^{2}\right)}\right)} \\
& =\frac{\widehat{\Delta}\left(q^{2}\right)}{1+R\left(q^{2}\right) \widehat{\Delta}\left(q^{2}\right)},
\end{aligned}
$$

\footnotetext{
${ }^{2}$ To go to Euclidean space, we set $q^{2}=-q_{\mathrm{E}}^{2}$, with $q_{\mathrm{E}}^{2}>0$ the positive square of a Euclidean four-vector, define the Euclidean propagator as $\Delta_{\mathrm{E}}\left(q_{\mathrm{E}}^{2}\right)=-\Delta\left(-q_{\mathrm{E}}^{2}\right)$, and the integration measure as $\int_{k}=i \int_{k_{\mathrm{E}}}$. To avoid notational clutter we always suppress the subscript "E".
} 


\begin{tabular}{|cccccc|c|}
\hline$\Delta(0)\left[\mathrm{GeV}^{-2}\right]$ & $\mu[\mathrm{GeV}]$ & $\alpha\left(\mu^{2}\right)$ & $m_{0}[\mathrm{MeV}]$ & $\alpha(0)$ & $R(0)$ & $\alpha_{\mathrm{PT}}(0)$ \\
\hline 5.51 & 2.5 & 0.467 & 600 & 6.40 & 0.31 & 0.47 \\
7.00 & 4.0 & 0.309 & 600 & 6.40 & 0.33 & 0.30 \\
5.51 & 2.5 & 0.467 & 500 & 4.45 & 0.15 & 0.62 \\
7.00 & 4.0 & 0.309 & 500 & 4.45 & 0.16 & 0.40 \\
\hline
\end{tabular}

Table 1. The gauge-invariant and universal IR fixed point $\alpha_{\mathrm{PT}}(0)$ obtained from the Landau gauge $\alpha(0)$ one after applying eq. (4.8).

arriving at the following relation for the two couplings,

$$
\alpha_{\mathrm{PT}}(0)=\frac{\alpha(0)}{1+R(0) \widehat{\Delta}(0)} .
$$

In order to get an approximate estimate for $\alpha_{\mathrm{PT}}(0)$ we need to determine the value of $R(0)$ from (4.6). To that end, we use the lattice data for the $\Delta(y)$ appearing on the rhs, and a mass $m^{2}(y)$ that displays power-law running, given by (2.5). The results of this procedure are summarized in table 1; clearly, the values obtained for $\alpha_{\mathrm{PT}}(0)$ are indeed much closer to the expectations based on phenomenological studies.

We emphasize that eq. (4.6) constitutes only a simplified estimate of the complete answer, and our results are suggestive at best. Note in particular that, as is evident from table 1, eq. (4.8) leads to the introduction of a spurious dependence on the renormalization scale $\mu$ for the ostensibly RG-invariant quantity $\alpha_{\mathrm{PT}}\left(q^{2}\right)$.

\section{Conclusions and outlook}

In this article we have shown how to extract effective QCD charges from the available (quenched) lattice data for some of the fundamental Green's functions of QCD. We use two different definitions of the effective charge, whose construction follows a similar procedure, relying on the construction of RG-invariant quantities out of the judicious combination of the various field-theoretic ingredients. The effective charges obtained display the characteristic feature of freezing at a common finite (non-vanishing) value in the deep IR, as expected from a variety of theoretical and phenomenological considerations.

In addition, we have offered a plausible explanation for the observed discrepancy in the freezing values of the effective charges obtained from the lattice and those derived from the fitting of various QCD processes, sensitive to non-perturbative physics. Our claim is that the underlying reason for the discrepancy is the difference in the gauges (Landau vs Feynman) used in the two approaches. We have studied this issue in the context of a toy model, which seems to corroborate this assertion.

It is clearly highly desirable to have available lattice results for the gluon and ghost propagators in gauges other than the Landau. In fact, a new gauge-fixing algorithm that may allow one to carry out lattice simulations in general $R_{\xi}$ gauges has been recently proposed [83]. In addition, it is of considerable theoretical importance to obtain lattice results in the Feynman gauge of the BFM [84], where, by virtue of the PT, quantities such 
as the gluon propagator acquire a gauge-invariant and universal status. Lattice results in this class of gauges would allow not only a direct determination of the phenomenologically relevant coupling $\alpha_{\mathrm{PT}}(0)$, but will furnish a stringent test of the SDE predictions for the gluon [73] and ghost propagators [85].

\section{Acknowledgments}

We would like to thank A. Cucchieri, T. Mendes, M. Müller-Preussker, and O. Oliveira for kindly making their lattice results available to us, and for their useful comments. The research of J. P. is supported by the European FEDER and Spanish MICINN under grant FPA2008-02878, and the Fundación General of the UV. The work of A.C.A is supported by the Brazilian Funding Agency CNPq under the grant 305850/2009-1.

\section{References}

[1] A. Cucchieri and T. Mendes, What's up with IR gluon and ghost propagators in Landau gauge? A puzzling answer from huge lattices, PoS (LATTICE 2007) 297 [arXiv:0710.0412] [SPIRES].

[2] A. Cucchieri and T. Mendes, Landau-gauge propagators in Yang-Mills theories at $\beta=0$ : massive solution versus conformal scaling, Phys. Rev. D 81 (2010) 016005 [arXiv: 0904.4033] [SPIRES].

[3] I.L. Bogolubsky, E.M. Ilgenfritz, M. Muller-Preussker and A. Sternbeck, The Landau gauge gluon and ghost propagators in $4 D S U(3)$ gluodynamics in large lattice volumes, PoS(LATTICE 2007) 290 [arXiv:0710.1968] [SPIRES].

[4] I.L. Bogolubsky, E.M. Ilgenfritz, M. Muller-Preussker and A. Sternbeck, Lattice gluodynamics computation of Landau gauge Green's functions in the deep infrared, Phys. Lett. B 676 (2009) 69 [arXiv:0901.0736] [SPIRES].

[5] O. Oliveira and P.J. Silva, The lattice infrared Landau gauge gluon propagator: the infinite volume limit, arXiv:0910.2897 [SPIRES].

[6] O. Oliveira and P.J. Silva, The lattice infrared Landau gauge gluon propagator: from finite volume to the infinite volume, arXiv:0911.1643 [SPIRES].

[7] J.M. Cornwall, Dynamical Mass Generation in Continuum QCD, Phys. Rev. D 26 (1982) 1453 [SPIRES].

[8] J.M. Cornwall and J. Papavassiliou, Gauge Invariant Three Gluon Vertex in QCD, Phys. Rev. D 40 (1989) 3474 [SPIRES].

[9] D. Binosi and J. Papavassiliou, Pinch Technique: Theory and Applications, Phys. Rept. 479 (2009) 1 [arXiv:0909.2536] [SPIRES].

[10] N.J. Watson, The gauge-independent QCD effective charge, Nucl. Phys. B 494 (1997) 388 [hep-ph/9606381] [SPIRES].

[11] D. Binosi and J. Papavassiliou, The QCD effective charge to all orders, Nucl. Phys. Proc. Suppl. 121 (2003) 281 [hep-ph/0209016] [SPIRES].

[12] A. Pilaftsis, Generalized pinch technique and the background field method in general gauges, Nucl. Phys. B 487 (1997) 467 [hep-ph/9607451] [SPIRES]. 
[13] A. Denner, G. Weiglein and S. Dittmaier, Gauge invariance of green functions: Background field method versus pinch technique, Phys. Lett. B 333 (1994) 420 [hep-ph/9406204] [SPIRES].

[14] S. Hashimoto, J. Kodaira, Y. Yasui and K. Sasaki, The Background field method: Alternative way of deriving the pinch technique's results, Phys. Rev. D 50 (1994) 7066 [hep-ph/9406271] [SPIRES].

[15] J. Papavassiliou, On the connection between the pinch technique and the background field method, Phys. Rev. D 51 (1995) 856 [hep-ph/9410385] [SPIRES].

[16] D. Binosi and J. Papavassiliou, The pinch technique to all orders, Phys. Rev. D 66 (2002) 111901 [hep-ph/0208189] [SPIRES].

[17] D. Binosi and J. Papavassiliou, Pinch technique self-energies and vertices to all orders in perturbation theory, J. Phys. G 30 (2004) 203 [hep-ph/0301096] [SPIRES].

[18] L.F. Abbott, The Background Field Method Beyond One Loop, Nucl. Phys. B 185 (1981) 189 [SPIRES].

[19] J.C. Taylor, Ward Identities and Charge Renormalization of the Yang-Mills Field, Nucl. Phys. B 33 (1971) 436 [SPIRES].

[20] W.J. Marciano and H. Pagels, Quantum Chromodynamics: A Review, Phys. Rept. 36 (1978) 137 [SPIRES].

[21] R. Alkofer, C.S. Fischer and F.J. Llanes-Estrada, Vertex functions and infrared fixed point in Landau gauge SU(N) Yang-Mills theory, Phys. Lett. B 611 (2005) 279 [Erratum ibid. 670 (2009) 460] [hep-th/0412330] [SPIRES].

[22] P.A. Grassi, T. Hurth and M. Steinhauser, Practical algebraic renormalization, Annals Phys. 288 (2001) 197 [hep-ph/9907426] [SPIRES].

[23] D. Binosi and J. Papavassiliou, Pinch technique and the Batalin-Vilkovisky formalism, Phys. Rev. D 66 (2002) 025024 [hep-ph/0204128] [SPIRES].

[24] D. Binosi and J. Papavassiliou, Gauge-invariant truncation scheme for the Schwinger-Dyson equations of QCD, Phys. Rev. D 77 (2008) 061702 [arXiv:0712.2707] [SPIRES].

[25] D. Binosi and J. Papavassiliou, New Schwinger-Dyson equations for non-Abelian gauge theories, JHEP 11 (2008) 063 [arXiv:0805.3994] [SPIRES].

[26] T. Kugo and I. Ojima, Local Covariant Operator Formalism of Nonabelian Gauge Theories and Quark Confinement Problem, Prog. Theor. Phys. Suppl. 66 (1979) 1 [SPIRES].

[27] A.C. Aguilar, D. Binosi, J. Papavassiliou and J. Rodriguez-Quintero, Non-perturbative comparison of QCD effective charges, Phys. Rev. D 80 (2009) 085018 [arXiv:0906.2633] [SPIRES].

[28] A.C. Mattingly and P.M. Stevenson, Optimization of $R\left(e+e^{-}\right)$and 'freezing' of the $Q C D$ couplant at low-energies, Phys. Rev. D 49 (1994) 437 [hep-ph/9307266] [SPIRES].

[29] Y.L. Dokshitzer, G. Marchesini and B.R. Webber, Dispersive Approach to Power-Behaved Contributions in QCD Hard Processes, Nucl. Phys. B 469 (1996) 93 [hep-ph/9512336] [SPIRES].

[30] L. von Smekal, R. Alkofer and A. Hauck, The infrared behavior of gluon and ghost propagators in Landau gauge QCD, Phys. Rev. Lett. 79 (1997) 3591 [hep-ph/9705242] [SPIRES]. 
[31] A.M. Badalian and V.L. Morgunov, Determination of $\alpha_{s}(1 \mathrm{GeV})$ from the charmonium fine structure, Phys. Rev. D 60 (1999) 116008 [hep-ph/9901430] [SPIRES].

[32] A.C. Aguilar, A.A. Natale and P.S. Rodrigues da Silva, Relating a gluon mass scale to an infrared fixed point in pure gauge QCD, Phys. Rev. Lett. 90 (2003) 152001 [hep-ph/0212105] [SPIRES].

[33] S.J. Brodsky, S. Menke, C. Merino and J. Rathsman, On the Behavior of the Effective QCD Coupling $\alpha_{\tau(s)}$ at Low Scales, Phys. Rev. D 67 (2003) 055008 [hep-ph/0212078] [SPIRES].

[34] S.J. Brodsky, New perspectives for QCD: The novel effects of final-state interactions and near-conformal effective couplings, Fizika B 13 (2004) 91 [hep-ph/0310289] [SPIRES].

[35] M. Baldicchi and G.M. Prosperi, Infrared behavior of the running coupling constant and bound states in QCD, Phys. Rev. D 66 (2002) 074008 [hep-ph/0202172] [SPIRES].

[36] G. Grunberg, Renormalization Scheme Independent QCD and QED: The Method of Effective Charges, Phys. Rev. D 29 (1984) 2315 [SPIRES].

[37] G. Grunberg, Evidence for infrared finite coupling in Sudakov resummation, Phys. Rev. D 73 (2006) 091901 [hep-ph/0603135] [SPIRES].

[38] H. Gies, Running coupling in Yang-Mills theory: A flow equation study, Phys. Rev. D 66 (2002) 025006 [hep-th/0202207] [SPIRES].

[39] D.V. Shirkov and I.L. Solovtsov, Analytic model for the QCD running coupling with universal $\alpha_{s}(0)$ value, Phys. Rev. Lett. 79 (1997) 1209 [hep-ph/9704333] [SPIRES].

[40] J.A. Gracey, One loop gluon form factor and freezing of $\alpha_{s}$ in the Gribov-Zwanziger QCD Lagrangian, JHEP 05 (2006) 052 [Erratum ibid. 1002 (2010) 078] [hep-ph/0605077] [SPIRES].

[41] G.M. Prosperi, M. Raciti and C. Simolo, On the running coupling constant in QCD, Prog. Part. Nucl. Phys. 58 (2007) 387 [hep-ph/0607209] [SPIRES].

[42] S.J. Brodsky and G.F. de Teramond, Light-front hadron dynamics and AdS/CFT correspondence, Phys. Lett. B 582 (2004) 211 [hep-th/0310227] [SPIRES].

[43] S.J. Brodsky, G.F. de Teramond and A. Deur, Nonperturbative QCD Coupling and its $\beta$ function from Light-Front Holography, Phys. Rev. D 81 (2010) 096010 [arXiv:1002.3948] [SPIRES].

[44] A.C. Aguilar, D. Binosi and J. Papavassiliou, Gluon and ghost propagators in the Landau gauge: Deriving lattice results from Schwinger-Dyson equations,

Phys. Rev. D 78 (2008) 025010 [arXiv:0802.1870] [SPIRES].

[45] F.V. Gubarev, L. Stodolsky and V.I. Zakharov, On the significance of the quantity $A^{2}$, Phys. Rev. Lett. 86 (2001) 2220 [hep-ph/0010057] [SPIRES].

[46] P. Boucaud et al., Testing Landau gauge OPE on the lattice with $a\left\langle A^{2}\right\rangle$ condensate, Phys. Rev. D 63 (2001) 114003 [hep-ph/0101302] [SPIRES].

[47] F.V. Gubarev and V.I. Zakharov, Emerging phenomenology of $\left\langle A_{\min }^{2}>\right.$, Phys. Lett. B 501 (2001) 28 [hep-ph/0010096] [SPIRES].

[48] J.A. Gracey, One loop renormalization of the non-local gauge invariant operator $\min _{\{U\}} \int d^{4} x\left(A_{\mu}^{a U}\right)^{2}$ in $Q C D$, Phys. Lett. B 651 (2007) 253 [arXiv:0706.1440] [SPIRES]. 
[49] M. Lavelle, Gauge invariant effective gluon mass from the operator product expansion, Phys. Rev. D 44 (1991) 26 [SPIRES].

[50] A.C. Aguilar and J. Papavassiliou, Power-law running of the effective gluon mass, Eur. Phys. J. A 35 (2008) 189 [arXiv:0708.4320] [SPIRES].

[51] D. Dudal, J.A. Gracey, S.P. Sorella, N. Vandersickel and H. Verschelde, A refinement of the Gribov-Zwanziger approach in the Landau gauge: infrared propagators in harmony with the lattice results, Phys. Rev. D 78 (2008) 065047 [arXiv:0806.4348] [SPIRES].

[52] C.W. Bernard, Monte Carlo Evaluation of the Effective Gluon Mass, Phys. Lett. B 108 (1982) 431 [SPIRES].

[53] C.W. Bernard, Adjoint Wilson lines and the effective gluon mass, Nucl. Phys. B 219 (1983) 341 [SPIRES].

[54] T. Iritani, H. Suganuma and H. Iida, Gluon-propagator functional form in the Landau gauge in SU(3) lattice QCD: Yukawa-type gluon propagator and anomalous gluon spectral function, Phys. Rev. D 80 (2009) 114505 [arXiv:0908.1311] [SPIRES].

[55] G. Parisi and R. Petronzio, On Low-Energy Tests of QCD, Phys. Lett. B 94 (1980) 51 [SPIRES].

[56] F. Halzen, G.I. Krein and A.A. Natale, Relating the QCD Pomeron to an effective gluon mass, Phys. Rev. D 47 (1993) 295 [SPIRES].

[57] F.J. Yndurain, Limits on the mass of the gluon, Phys. Lett. B 345 (1995) 524 [SPIRES].

[58] A. Szczepaniak, E.S. Swanson, C.-R. Ji and S.R. Cotanch, Glueball Spectroscopy in a Relativistic Many-Body Approach to Hadron Structure, Phys. Rev. Lett. 76 (1996) 2011 [hep-ph/9511422] [SPIRES].

[59] J.H. Field, A phenomenological analysis of gluon mass effects in inclusive radiative decays of the $J / \psi$ and Upsilon, Phys. Rev. D 66 (2002) 013013 [hep-ph/0101158] [SPIRES].

[60] A.C. Aguilar, A. Mihara and A.A. Natale, Freezing of the QCD coupling constant and solutions of Schwinger-Dyson equations, Phys. Rev. D 65 (2002) 054011 [hep-ph/0109223] [SPIRES].

[61] E.G.S. Luna, A.F. Martini, M.J. Menon, A. Mihara and A.A. Natale, Influence of a dynamical gluon mass in the $p p$ and $\bar{p} p$ forward scattering, Phys. Rev. D 72 (2005) 034019 [hep-ph/0507057] [SPIRES].

[62] E.G.S. Luna, Survival probability of large rapidity gaps in a QCD model with a dynamical infrared mass scale, Phys. Lett. B 641 (2006) 171 [hep-ph/0608091] [SPIRES].

[63] O. Oliveira and P. Bicudo, Running Gluon Mass from Landau Gauge Lattice QCD Propagator, arXiv:1002.4151 [SPIRES].

[64] D. Dudal, O. Oliveira and N. Vandersickel, Indirect lattice evidence for the Refined Gribov-Zwanziger formalism and the gluon condensate $\left\langle A^{2}\right\rangle$ in the Landau gauge, Phys. Rev. D 81 (2010) 074505 [arXiv: 1002 .2374] [SPIRES].

[65] J.S. Ball and T.-W. Chiu, Analytic properties of the vertex function in gauge theories. 2, Phys. Rev. D 22 (1980) 2550 [Erratum ibid. D 23 (1981) 3085]. [SPIRES].

[66] T. Kugo, The universal renormalization factors $Z(1) / Z(3)$ and color confinement condition in non-Abelian gauge theory, hep-th/9511033 [SPIRES]. 
[67] P.A. Grassi, T. Hurth and A. Quadri, On the Landau background gauge fixing and the IR properties of YM Green functions, Phys. Rev. D 70 (2004) 105014 [hep-th/0405104] [SPIRES].

[68] K.-I. Kondo, Kugo-Ojima color confinement criterion and Gribov-Zwanziger horizon condition, Phys. Lett. B 678 (2009) 322 [arXiv:0904.4897] [SPIRES].

[69] H. Nakajima and S. Furui, Test of the Kugo-Ojima confinement criterion in the lattice Landau gauge, Nucl. Phys. Proc. Suppl. 83 (2000) 521 [hep-lat/9909008] [SPIRES].

[70] H. Nakajima and S. Furui, Numerical study of lattice Landau gauge QCD and the Gribov copy problem, Nucl. Phys. Proc. Suppl. 141 (2005) 34 [hep-lat/0408001] [SPIRES].

[71] S. Furui and H. Nakajima, Infrared features of unquenched finite temperature lattice Landau gauge QCD, Phys. Rev. D 76 (2007) 054509 [hep-lat/0612009] [SPIRES].

[72] A. Sternbeck, The infrared behavior of lattice QCD Green's functions, hep-lat/0609016 [SPIRES].

[73] A.C. Aguilar, D. Binosi and J. Papavassiliou, Indirect determination of the Kugo-Ojima function from lattice data, JHEP 11 (2009) 066 [arXiv:0907.0153] [SPIRES].

[74] A. Cucchieri, T. Mendes and A. Mihara, Numerical study of the ghost-gluon vertex in Landau gauge, JHEP 12 (2004) 012 [hep-lat/0408034] [SPIRES].

[75] E.M. Ilgenfritz, M. Muller-Preussker, A. Sternbeck and A. Schiller, Gauge-variant propagators and the running coupling from lattice QCD, hep-lat/0601027 [SPIRES].

[76] P. Boucaud et al., Artefacts and $<A^{2}>$ power corrections: Reexamining $Z_{\psi}\left(p^{2}\right)$ and $Z_{V}$, Phys. Rev. D 74 (2006) 034505 [hep-lat/0504017] [SPIRES].

[77] P. Boucaud et al., Ghost-gluon running coupling, power corrections and the determination of $\Lambda_{\overline{\mathrm{MS}}}$, Phys. Rev. D 79 (2009) 014508 [arXiv:0811.2059] [SPIRES].

[78] P. Boucaud et al., Gribov's horizon and the ghost dressing function, Phys. Rev. D 80 (2009) 094501 [arXiv: 0909. 2615] [SPIRES].

[79] J.M. Cornwall, Positivity issues for the pinch-technique gluon propagator and their resolution, Phys. Rev. D 80 (2009) 096001 [arXiv: 0904.3758] [SPIRES].

[80] G. Weiglein, Gauge dependence of Green functions and algebraic calculation of general two loop selfenergies, Diploma Thesis, University of Würzburg (1994).

[81] G. Degrassi and A. Sirlin, Gauge invariant selfenergies and vertex parts of the Standard Model in the pinch technique framework, Phys. Rev. D 46 (1992) 3104 [SPIRES].

[82] J. Papavassiliou, Gauge independent transverse and longitudinal self energies and vertices via the pinch technique, Phys. Rev. D 50 (1994) 5958 [hep-ph/9406258] [SPIRES].

[83] A. Cucchieri, T. Mendes and E.M.S. Santos, Covariant gauge on the lattice: a new implementation, Phys. Rev. Lett. 103 (2009) 141602 [arXiv:0907.4138] [SPIRES].

[84] R.F. Dashen and D.J. Gross, The Relationship Between Lattice and Continuum Definitions of the Gauge Theory Coupling, Phys. Rev. D 23 (1981) 2340 [SPIRES].

[85] A.C. Aguilar and J. Papavassiliou, Infrared finite ghost propagator in the Feynman gauge, Phys. Rev. D 77 (2008) 125022 [arXiv:0712.0780] [SPIRES]. 